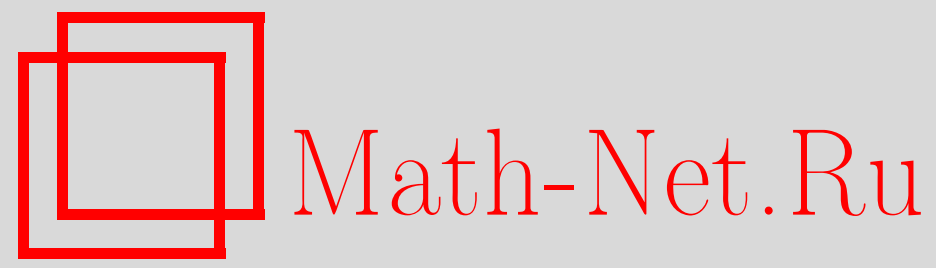

Общероссийский математический портал

С. А. Гриценко, О нулях специального вида функций, связанных с рядами Дирихле из класса Сельберга, Матем. заметки, 1996, том 60, выпуск 4, 601-605

DOI: https://doi.org/10.4213/mzm1868

Использование Общероссийского математического портала MathNet.Ru подразумевает, что вы прочитали и согласны с пользовательским соглашением

http://www.mathnet.ru/rus/agreement

Параметры загрузки: 
IP: 52.6 .47 .48

26 апреля 2023 г., 17:49:41

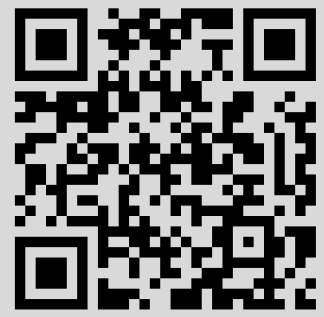




\section{О НУЛЯХ СПЕЦИАЛЬНОГО ВИДА ФУНКЦИЙ, СВЯЗАННЫХ С РЯДАМИ ДИРИХЛЕ \\ ИЗ КЛАССА СЕЛЬБЕРГА}

\section{С. А. Гриценко}

А. Сельберг определил в [1] класс $S$ рядов Дирихле

$$
F(s)=\sum_{n=1}^{\infty} a(n) n^{-s}, \quad \operatorname{Re}(s)>1,
$$

удовлетворяющих следующим условиям:

1) существует целое неотрицательное число $m$ такое, что функция $(s-1)^{m} F(s)$ целая;

2) коэффициенты Дирихле $a(n)$ удовлетворяют неравенствам

$$
a(n) \ll_{\varepsilon} n^{\varepsilon}
$$

для любого положительного $\varepsilon$, причем, $a(1)=1$;

$3)$ существует функция $\gamma_{F}(s)$ вида

$$
\gamma_{F}(s)=\varepsilon_{1} Q^{s} \prod_{\nu=1}^{k} \Gamma\left(\lambda_{\nu} s+\mu_{\nu}\right)
$$

где $\left|\varepsilon_{1}\right|=1, k=k_{F}$ - натуральное число, $Q>0, \lambda_{\nu}>0, \operatorname{Re}\left(\mu_{\nu}\right) \geqslant 0$, и такая, что для функции $\Phi(s)=\gamma_{F}(s) F(s)$ справедливо тождество

$$
\Phi(s)=\bar{\Phi}(1-\bar{s}) ;
$$

Работа вьполненапри финансовой поддержке Российского фонда фундаментальных исследований, грант № 94-01-00002. 
4) при $\operatorname{Re}(s)>1$ функция $F(s)$ раскладьвается в эйлерово произведение

$$
F(s)=\prod_{p}\left(1+a(p) p^{-s}+a\left(p^{2}\right) p^{-2 s}+\cdots\right),
$$

$p$ пробегает простые числа;

$$
\log F(s)=\sum_{n=1}^{\infty} b(n) n^{-s}
$$

где $b(n)=0$, если $n$ не равно положительной степени простого числа, причем, $b(n) \ll n^{\theta}$ для некоторого $\theta \leqslant 1 / 2$.

В статье [2] для любой функции $F(s)$ из $S$ определена степень $F(s)$ следующим образом:

$$
d_{F}=2 \sum_{\nu=1}^{k} \lambda_{\nu}
$$

Изложим известные факты и гипотезы о распределении нулей рядов Дирихле (см. также [3]-[6]).

Пусть $F(s)$ - функция из класса $S$. Назовем нули $F(s)$, совпадающие с полюсами функции $\gamma_{F}(s)$, тривиальнылми, а остальные - нетривиальными.

Известно (см. [1]), что нетривиальные нули $F(s)$ расположены в полосе $1-A \leqslant$ $\operatorname{Re}(s) \leqslant A, A=A_{F}>0$.

Кроме того, так как $F(s)$ удовлетворяет функциональному уравнению риманова типа, то

$$
N(T) \sim c T \log T, \quad c=c_{F}>0,
$$

где $N(T)$ - число нетривиальных нулей $\rho$ функции $F(s)$ таких, что $0<\operatorname{Im}(\rho) \leqslant T$.

Будем говорить, что для $F(s)$ справедлива гипотеза Римана, если все ее нетривиальные нули лежат на прямой $\operatorname{Re}(s)=1 / 2$.

Известно, что существуют функции, представляющиеся в области $\operatorname{Re}(s)>1$ рядами Дирихле, удовлетворяющие функциональньп уравнениям риманова типа, но не имеющие разложений в эйлеровы произведения, для которых гипотеза Римана неверна. Таковы, например, функция Дэвенпорта-Хейльбронна (см. [7], [8], [3]), дзета-функции Эпштейна, соответствующие неодноклассным бинарным квадратичньм формам (см. [9], [10], [5], [6]) и дзета-функция Гурвица (см. [12], [14]). Все перечисленные функции представляются в виде линейных комбинаций рядов Дирихле, имеющих функциональное уравнение и разложение в эйлерово произведение.

Следующие гипотезы, принадлежащие соответственно А.Сельбергу [1], А.А. Карацубе [3] и Г. Монтгомери (см. [1, с. 384]), пока не доказаны и не опровергнуты:

для всех функций из класса $S$ (которые по определению не только удовлетворяют функциональным уравнениям риманова типа, но и имеют разложения в эйлеровы произведения), справедлива гипотеза Римана;

почти все нетривиальные нули функции Дэвенпорта-Хейльбронна лежат на прямой $\operatorname{Re}(s)=1 / 2$;

почти все нетривиалные нули дзета-функций Эпштейна лежат на прямой $\operatorname{Re}(s)=1 / 2$. 
Э. Бомбьери и Д. Хейчал дали в [5] условное доказательство гипотезы Монтгомери.

Введем три функции:

$$
\begin{gathered}
\rho(s)=\bar{\gamma}_{F}(1-\bar{s}) \gamma_{F}^{-1}(s)=\bar{\varepsilon}_{1}^{2} Q^{1-2 s} \prod_{\nu=1}^{k} \Gamma\left(\lambda_{\nu}(1-s)+\bar{\mu}_{\nu}\right) \Gamma^{-1}\left(\lambda_{\nu}+\mu_{\nu}\right), \\
\exp (i \theta(t))=\left(\rho\left(\frac{1}{2}+i t\right)\right)^{-1 / 2}, \quad Z(t)=\exp (i \theta(t)) F\left(\frac{1}{2}+i t\right) .
\end{gathered}
$$

Из функционального уравнения следует, что $Z(t)$ принимает вещественные значения при вещественных $t$.

Пусть теперь $a_{1}, \ldots, a_{N}$-произвольные вещественные числа. Рассмотрим фуункцию $\Phi_{1}(s)$ вида

$$
\Phi_{1}(s)=\sum_{j=1}^{N} a_{j}\left(\rho_{j}(s)\right)^{-1 / 2} F_{j}(s)
$$

где $F_{j}(s)-$ функции из $S$.

Определим функцию $f(t)$ :

$$
f(t)=\Phi_{1}\left(\frac{1}{2}+i t\right)=\sum_{j=1}^{N} a_{j}\left(\rho_{j}\left(\frac{1}{2}+i t\right)\right)^{-1 / 2} F_{j}\left(\frac{1}{2}+i t\right)=\sum_{j=1}^{N} a_{j} Z_{j}(t) .
$$

Начиная отсюда будем считать, что $T \geqslant T_{0}>0$, где $T_{0}-$ достаточно большая постоянная, зависящая лишш от $F_{1}(s), \ldots, F_{N}(s)$.

Как отмечено в [3], $\Phi_{1}(s)$ "подобна" функции Дэвенпорта-Хейльбронна (все $F_{j}(s)$ удовлетворяют функциональным уравнениям риманова типа, а $\Phi_{1}(s)$, вообще говоря, не имеет разложения в эйлерово произведение).

Если для $\Phi_{1}(s)$ справедлива гипотеза, сформулированная для функции Дэвенпорта-Хейльбронна (в связи с этим см. $\left[1\right.$, п. 4]), то существует постоянная $c_{1}=$ $c_{1}\left(\Phi_{1}\right)>0$ такая, что

$$
N_{0}(T) \geqslant c_{1} T \log T .
$$

Назовем последнее неравенство "ожидаемым".

Для случая, когда $F_{1}(s), \ldots, F_{N}(s)$ являются $L$-функциями Дирихле, отвечающими примитивным характерам (в нашей терминологии они относятся к функциям из класса $S$ степени 1 ), А. А. Карацуба получил в [3] своим методом следующие оценки величины $N_{0}(T)$

$$
N_{0}(T) \geqslant T(\log T)^{2 \beta} \exp \left(-c_{2} \sqrt{\log \log T}\right),
$$

если характеры Дирихле $\chi_{1}, \ldots, \chi_{N}$ имеют одинаковую четность,

$$
N_{0}(T) \geqslant T(\log T)^{\beta} \exp \left(-c_{3} \sqrt{\log \log T}\right)
$$

- в противном случае; здесь $\beta=(\varphi(K))^{-1}, K$ - наименьшее общее кратное натуральных чисел $k_{1}, \ldots, k_{N}$ - модулей характеров $\chi_{1}, \ldots, \chi_{N}, \varphi(K)$ - функция Эйлера, $c_{2}, c_{3}$ - абсолютные положительные постоянные. 
В настоящей статье анонсируется оценка снизу величины $N_{0}(T)$ для случая, когда $F_{1}(s), \ldots, F_{N}(s)$ - функции из $S$ степени 2 .

Для $F_{1}(s), \ldots, F_{N}(s)$ принимается ряд гипотез, формулировки которых приводятся ниже.

Прежде всего заметим, что если в условиях нашей теоремы применить метод Харди-Литтлвуда [11], то получится неравенство

$$
N_{0}(T) \geqslant c_{4} T, \quad c_{4}>0
$$

и задача состоит в его уточнении.

Из нашей теоремы легко вывести, что

$$
N_{0}(T) \geqslant \frac{1}{2} T \exp (\sqrt{\log \log \log T}) .
$$

Эта оценка, хотя из нее следует, что прямая $\operatorname{Re}(s)=1 / 2$ является “особой" для нулей функции $\Phi_{1}(s)$, очевидно, гораздо менее точна, чем выписанные вьше оценки А. А. Карацубы, и далека от "ожидаемой”.

Первый результат о том, что прямая $\operatorname{Re}(s)=1 / 2$ является "особой" для нулей функции Дэвенпорта-Хейльбронна был получен в 1980 году С. М. Ворониньп [13]. Им было доказано, что

$$
N_{0}(T) \geqslant c_{5} T \exp \left(\frac{1}{20} \sqrt{\log \log \log \log T}\right)
$$

( $c_{5}$ - абсолютная положительная постоянная).

Переходим к формулировке условий для функций $F_{1}(s), \ldots, F_{N}(s)$ (в связи с этим см. также статью [1]). Пусть $F_{1}(s), \ldots, F_{N}(s)$ - функции из $S$ степени 2 . Пусть, кроме того, они таковы, что

1) при $x \rightarrow \infty$ справедливы асимптотические формулы

$$
\sum_{p \leqslant x}\left|a_{j}(p)\right|^{2} \log p=x+O\left(x(\log x)^{-1}\right),
$$

где $a_{j}(n)$ - коэффициенты Дирихле функций $F_{j}(s)$, а $p$ здесь и везде в дальнейшем обозначает простые числа;

2) при $x \rightarrow \infty$ справедливы оценки

$$
\sum_{p \leqslant x} a_{j}(p) \bar{a}_{i}(p) p^{-1}=O(1),
$$

где $a_{j}(n)$ и $a_{i}(n)$ - коэффициенты Дирихле любых функций $F_{j}(s), F_{i}(s)$, $1 \leqslant j \neq i \leqslant N$

3) при любых $j, 1 \leqslant j \leqslant N$, существуют положительные постоянные $c_{F_{j}}$ такие, что

$$
\left|1+a_{j}(p) p^{-1 / 2-i t}+a_{j}\left(p^{2}\right) p^{-1-2 i t}+\cdots\right|>c_{F_{j}} ;
$$

$4)$ пусть $F(s)$ - любая из функций $F_{1}(s), \ldots, F_{N}(s)$; тогда при $x \rightarrow \infty$ справедлива асимптотическая формула

$$
\sum_{n \leqslant x}|a(n)|^{2} n^{-1}=A_{F} \log x+B_{F}+O\left((\log x)^{-4}\right),
$$

где $a(n)$ - коэффициенты Дирихле функции $F(s), A_{F}>0, B_{F}$ - постоянные, зависящие только от $F(s)$.

Сформулируем наш основной результат. 
Теорема. Пусть $N$ - произвольное натуральное число, $a_{1}, \ldots, a_{N}-$ произвольные вещественные числа, $a_{1} \neq 0, T \geqslant T_{0}>0$. Пусть $f(t)$ - опреде-

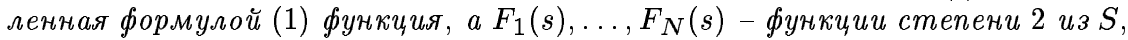
удовлетворяющие условиям 1-4.

Тогда на отрезке $[T, 2 T]$ лехит не меньше, чем $T \exp (\sqrt{\log \log \log T})$, нулей нечетного порядка функции $f(t)$.

ДокАЗАТЕ ЛЬСтво теоремы проводится методом С. М. Воронина (см. [13], [12]), разработанным им в 1980 году для оценки числа нулей функции Дэвенпорта-Хейльбронна, лежащих на отрезке критической прямой.

ЗАмЕч АниЕ. Можно указать подкласс класса $S$, все функции которого удовлетворяют условиям 1-4. Его составляют различные $L$-функции Гекке, понимаемые в том же смысле, что в монографии [12], и соответствующие комплексным характерам Гекке одного и того же мнимого квадратичного расширения поля рациональных чисел. Для функций данного подкласса утверждение теоремы безусловно.

Математический институт

Поступило им. В.А. Стеклова РАН 23.05 .96

\section{СПИСОК ЦИТИРОВАННОЙ ЛИТЕРАТУРЫ}

1. Selberg A. // Proc. of the Amalfi conference on Analytic Number Theory: Univ. di Salerno, 1992. P. 365-387. 2. Conrey J. B., Ghosh A. // Duke Math. J. 1993. V. 72. № 3. Р. 673-695. 3. Карацуба А. А. // Тр. МИРАН. 1994. Т. 207. C. 180-196. 4. Карацуба A. A. // Math. Slovaca. 1994. T. 44. № 5. C. 633-649. 5. Bombieri E., Hejhal D. // C. R. Acad. Sci. Paris. Ser. A. 1987. V. 304. P. 213-217. 6. Hejhal D. // Proc. International Congress Math. Berkeley. California, 1986. P. 1362-1383. 7. Davenport H., Heilbronn H. // J. London Math. Soc. 1936. V. 11. Р. 181-185, 307-312. 8. Карацуба А. А. // Изв. АН СССР. Сер. матем. 1990. Т. 54. № 2. С. 303-315. 9. Воронин С. М. // Тр. МИАН. 1976. Т. 142. С. 136-147. 10. Воронин С. М. // Докл. АН СССР. 1977. Т. 235. №2. С. 257-258. 11. Hardy G., Littlewood J. E. // Math. Zs. 1921. V. 10. P. 283-317. 12. Boронин С. М., Карацуба А. А. Дзета-функция Римана. М.: Физматлит, 1994. 13. Воронин С. М. // Изв. АН СССР. Сер. матем. 1980. Т. 44. №1. С. 63-91. 14. Титчмарш Е. К. Теория дзета-функции Римана. М.: ИЛ, 1953. 